\title{
Late Stage Intermolecular CH Activation for Lead Diversification: A Highly Chemoselective Oxyfunctionalization of the C-9 Position of Potent Bryostatin Analogues
}

Paul A. Wender,* Michael K. Hilinski, and Alexander V. W. Mayweg

Department of Chemistry, Stanford University, Stanford, CA 94305-5080

Supporting Information

General Methods. Air- and moisture-sensitive reactions were carried out in oven-dried glassware sealed with rubber septa under a positive pressure of dry nitrogen or argon from a manifold or balloon, unless otherwise indicated. Similarly sensitive liquids and solutions were transferred via syringe or stainless steel cannula. Reactions were stirred using Teflon-coated magnetic stir bars. Organic solutions were concentrated using a Buchi rotary evaporator with a teflon seal vacuum pump. Analytical TLC was performed with $0.25 \mathrm{~mm}$ silica gel $60 \mathrm{~F}$ plates with $254 \mathrm{~nm}$ fluorescent indicator from Merck. Plates were visualized by ultraviolet light and treatment with acidic $p$-anisaldehyde stain followed by gentle heating. Chromatographic purification of products was accomplished by flash chromatography, as described by Still and coworkers, ${ }^{1}$ unless otherwise indicated. Silica gel 60, 230-400 mesh was purchased from EM.

NMR spectra were measured on a Varian INOVA $500(1 \mathrm{H}$ at $500 \mathrm{MHz}, 13 \mathrm{C}$ at 125 $\mathrm{MHz})$, or Varian INOVA $600(1 \mathrm{H}$ at $600 \mathrm{MHz}, 13 \mathrm{C}$ at $150 \mathrm{MHz})$ magnetic resonance spectrometer. Data for 1H NMR spectra are reported as follows: chemical shift (d ppm), integration, multiplicity $(\mathrm{s}=$ singlet, brs $=$ broad singlet, $\mathrm{d}=$ doublet, brd $=$ broad doublet, $\mathrm{t}=$ triplet, $\mathrm{q}=$ quartet, $\mathrm{dd}=$ doublet of doublets, $\mathrm{dt}=$ doublet of triplets, $\mathrm{ddd}=$ doublet of doublet of doublets, dddd $=$ doublet of doublet of doublet of doublets, $\mathrm{ddt}=$ doublet of doublet of triplets, $\mathrm{m}$ $=$ multiplet), coupling constant $(\mathrm{Hz})$. Data for $13 \mathrm{C}$ are reported in terms of chemical shift relative to residual solvent peak. Infrared spectra were recorded on a Perkin-Elmer 1600 Series Fourier transform spectrometer (FTIR) and are reported in wavenumbers $\left(\mathrm{cm}^{-1}\right)$. High resolution mass spectra (HRMS) were recorded at the High Resolution Mass Spectrometry Facility, University of California at Riverside. Purity is documented via high field ${ }^{1} \mathrm{H}$ NMR spectra showing at most only trace peaks not attributable to the assigned structure.
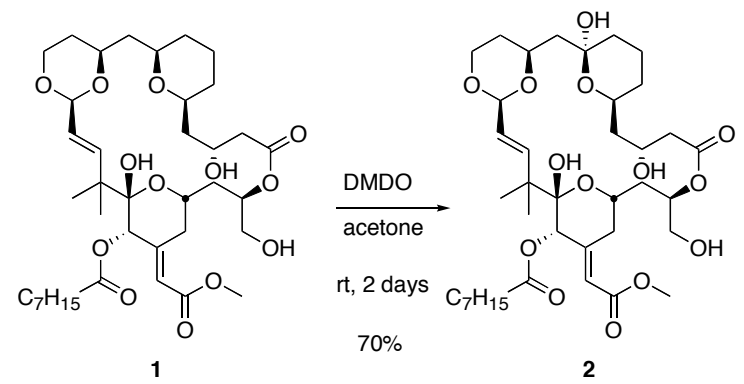

To a solution of \# (3 mg, $4.14 \mu \mathrm{mol}, 1$ eq.) in a disposable glass vial equipped with a stir bar was added freshly prepared and distilled DMDO as solution in acetone $(8.3 \mu \mathrm{mol}, 2 \mathrm{eq}$.). The reaction was sealed with PTFE tape, screw-capped, and stirred at ambient temperature for 24 hours. Additional DMDO in acetone (8.3 $\mu \mathrm{mol}, 2$ eq. $)$ was added and the vial was resealed and stirred an additional 24 hours. The volatiles were evaporated under a stream of $\mathrm{N}_{2}$ and the crude material was purified via HPLC (C-18 column, $\mathrm{MeCN} / \mathrm{H}_{2} \mathrm{O}$ eluent). The collected fractions were lyophilized to provide 9 -oxy product 2 (2 $\mathrm{mg}, 70 \%)$ as a white amorphous solid.

\footnotetext{
${ }^{1}$ Still, W.C.; Kahn, M.; Mitra, A. J. Org. Chem., 1978, 43, 2923.
} 


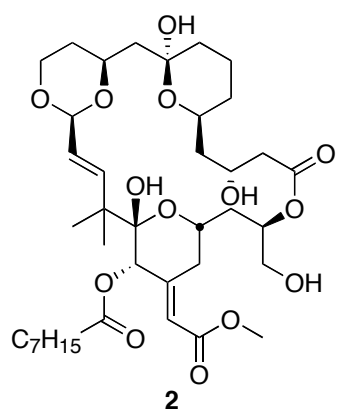

Data for 2:

$\mathbf{R} \boldsymbol{f}$ (4:1 ethyl acetate:petroleum ether) $=0.14$; one spot, brown ( $\mathrm{p}$-anisaldehyde stain).

Optical Rotation: $[\alpha]_{\mathrm{D}}^{22.5}=-10.9\left(\mathrm{c}=0.07, \mathrm{CDCl}_{3}\right)$.

$1 \mathrm{H}$ NMR $\left(600 \mathrm{MHz}, \mathrm{C}_{6} \mathrm{D}_{6}\right): \delta=6.44(\mathrm{~d}, J=16 \mathrm{~Hz}, 1 \mathrm{H}), 6.40(\mathrm{~d}, J=2 \mathrm{~Hz}, 1 \mathrm{H}), 5.84(\mathrm{dd}, J=16,7 \mathrm{~Hz}$, $1 \mathrm{H}), 5.68(\mathrm{~s}, 1 \mathrm{H}), 5.67(\mathrm{~s}, 1 \mathrm{H}), 5.49(\mathrm{~m}, 1 \mathrm{H}), 5.43(\mathrm{~d}, J=7 \mathrm{~Hz}, 1 \mathrm{H}), 4.57(\mathrm{~d}, J=12 \mathrm{~Hz}, 1 \mathrm{H}), 4.45$ (ap. t, $J$ $=11.5,2.5 \mathrm{~Hz}, 1 \mathrm{H}), 4.29(\mathrm{dd}, J=14,2 \mathrm{~Hz}, 1 \mathrm{H}), 4.10-4.05(\mathrm{~m}, 2 \mathrm{H}), 3.86(\mathrm{br} \mathrm{d}, J=9 \mathrm{~Hz}, 1 \mathrm{H}), 3.70-3.60(\mathrm{~m}$, $3 \mathrm{H}), 3.47(\mathrm{dd}, J=12,6 \mathrm{~Hz}, 1 \mathrm{H}), 3.18(\mathrm{~s}, 3 \mathrm{H}), 2.59$ (ap. t, $J=12 \mathrm{~Hz}, 1 \mathrm{H}), 2.34$ (ap. t, $J=14 \mathrm{~Hz}, 1 \mathrm{H}), 2.17$ (dd, $J=12.5,2.5 \mathrm{~Hz}, 1 \mathrm{H}), 2.09$ (m, 2H), 1.89 (br dd, $J=14,7 \mathrm{~Hz}, 1 \mathrm{H}), 1.71$ (ap. t, $J=13 \mathrm{~Hz}, 1 \mathrm{H}), 1.57-$ $1.48(\mathrm{~m}, 2 \mathrm{H}), 1.47(\mathrm{~s}, 3 \mathrm{H}), 1.43-1.35(\mathrm{~m}, 2 \mathrm{H}), 1.35(\mathrm{~s}, 3 \mathrm{H}), 1.23-1.07$ (br m, 16H), 0.91-0.84 (m, $2 \mathrm{H}), 0.85$ $(\mathrm{t}, J=7 \mathrm{~Hz}, 3 \mathrm{H}), 0.78-0.74(\mathrm{~m}, 2 \mathrm{H}) \mathrm{ppm}$.

${ }^{13}$ C-NMR $\left(150 \mathrm{MHz}, \mathrm{C}_{6} \mathrm{D}_{6}\right): \delta=172.61,171.48,166.81,152.36,142.39,126.67,120.44,103.12,99.62$, $95.58,74.71,71.64,70.44,68.64,67.43,66.04,65.69,65.02,50.43,49.06,45.48,42.00,39.49,36.16$, $34.91,34.60,32.82,31.85,31,63,30.82,29.14,29.11,24.93,24.77,22.81,19.54,18.31,14.17 \mathrm{ppm}$.

IR (FTIR, film) v = 3306, 2929, 2856, 1733, 1666, 1462, 1434, 1382, 1298, 1260, 1230, 1154, 1093, 1029 , $975 \mathrm{~cm}^{-1}$.

HRMS: Calculated for $\mathrm{C}_{38} \mathrm{H}_{60} \mathrm{O}_{14} \mathrm{Na}(\mathrm{M}+\mathrm{Na})^{+}:$763.3875, found: 763.3915 .
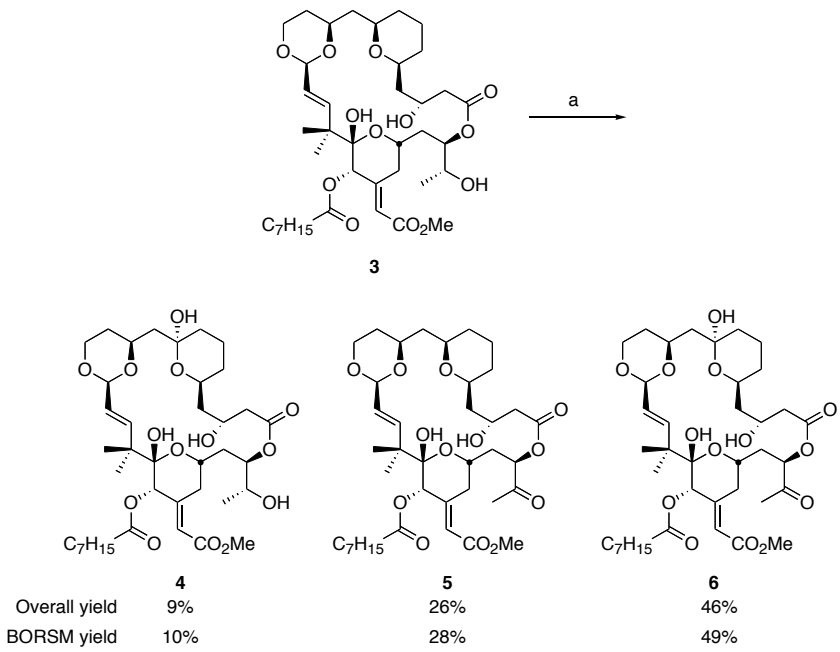

To a solution of $\mathbf{3}$ (3.4 mg, $4.60 \mu \mathrm{mol}$, 1eq.) in a disposable glass vial equipped with a stir bar was added freshly prepared and distilled DMDO as solution in acetone $(9.2 \mu \mathrm{mol}, 2 \mathrm{eq}$.$) . The reaction was sealed with$ PTFE tape, screw-capped, and stirred at ambient temperature for 24 hours. Additional DMDO in acetone (9.2 $\mu \mathrm{mol}, 2$ eq.) was added and the vial was resealed and stirred an additional 24 hours. The volatiles were evaporated under a stream of $\mathrm{N}_{2}$ and the crude material was purified via HPLC (C-18 column, $\mathrm{MeCN} / \mathrm{H}_{2} \mathrm{O}$ eluent). The collected fractions were lyophilized to provide 9 -oxy product $4(0.3 \mathrm{mg}, 9 \%, 10 \%$ borsm), ketone 5 (0.9 mg, 26\%, 28\% borsm), and 9-oxy ketone 6 (1.6 mg, 46\%, 49\% borsm) as white amorphous solids. 


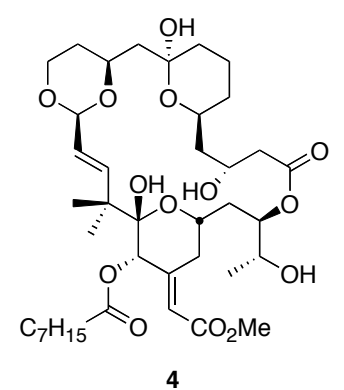

Data for 4:

$\mathbf{R} \boldsymbol{f}$ (4:1 ethyl acetate:petroleum ether) $=0.14$; one spot, brown (p-anisaldehyde stain).

Optical Rotation: $[\alpha]_{\mathrm{D}}^{22.5}=+14.5\left(\mathrm{c}=0.06, \mathrm{CDCl}_{3}\right)$.

$1 \mathrm{H}$ NMR $\left(600 \mathrm{MHz}, \mathrm{C}_{6} \mathrm{D}_{6}\right): \delta=6.50(\mathrm{~d}, J=16 \mathrm{~Hz}, 1 \mathrm{H}), 6.41(\mathrm{~d}, J=2 \mathrm{~Hz}, 1 \mathrm{H}), 5.85(\mathrm{dd}, J=16,7 \mathrm{~Hz}$, $1 \mathrm{H}), 5.77(\mathrm{~s}, 1 \mathrm{H}), 5.68(\mathrm{~s}, 1 \mathrm{H}), 5.51(\mathrm{~d}, J=7 \mathrm{~Hz}, 1 \mathrm{H}), 5.41(\mathrm{~m}, 1 \mathrm{H}), 4.58(\mathrm{~d}, J=12 \mathrm{~Hz}, 1 \mathrm{H}), 4.46(\mathrm{~m}, 2 \mathrm{H})$, $4.34(\mathrm{~d}, J=12 \mathrm{~Hz}, 1 \mathrm{H}), 4.10-4.05(\mathrm{~m}, 2 \mathrm{H}), 3.86(\mathrm{dd}, J=11,5 \mathrm{~Hz}, 1 \mathrm{H}), 3.71-3.66(\mathrm{~m}, 2 \mathrm{H}), 3.62($ ap. $\mathrm{t}, J=$ $11.5 \mathrm{~Hz}, 1 \mathrm{H}), 3.19$ (s, 3H), 2.57 (ap. t, $J=12 \mathrm{~Hz}, 1 \mathrm{H}), 2.40$ (ap. t, $J=12 \mathrm{~Hz}, 1 \mathrm{H}), 2.17(\mathrm{~d}, J=12 \mathrm{~Hz}, 1 \mathrm{H})$, 2.09-2.05 (m, 2H), $1.90(\mathrm{dd}, J=15,8 \mathrm{~Hz}, 1 \mathrm{H}), 1.77-1.74(\mathrm{~m}, 2 \mathrm{H}), 1.57-1.45(\mathrm{~m}, 3 \mathrm{H}), 1.48(\mathrm{~s}, 3 \mathrm{H}), 1.26(\mathrm{~s}$, $3 \mathrm{H}), 1.20-1.03$ (br m, 16H), 1.03 (d, $J=7 \mathrm{~Hz}, 3 \mathrm{H}), 0.91-0.84(\mathrm{~m}, 2 \mathrm{H}), 0.85$ (t, $J=7 \mathrm{~Hz}, 3 \mathrm{H}), 0.77-0.72$ (m, 2H) $\mathrm{ppm}$.

${ }^{13}$ C-NMR $\left(150 \mathrm{MHz}, \mathrm{C}_{6} \mathrm{D}_{6}\right): \delta=172.35,171.48,166.64,152.39,142.56,126.67,120.37,103.12,99.66$, $95.56,74.70,74.07,70.50,70.16,68.73,67.40,66.06,65.12,50.40,49.06,45.41,42.00,39.65,36.16$, $34.91,34.58,32.89,31.83,31.30,30.84,29.12,29.07,24.91,24.80,22.78,19.78,19.55,18.38,14.13$ ppm. IR (FTIR, film) v = 3306, 2924, 2850, 1738, 1721, 1666, 1650, 1633, 1462, 1434, 1381, 1298, 1260, 1153 , 1095, 1066, 1027, $975 \mathrm{~cm}^{-1}$.

HRMS: Calculated for $\mathrm{C}_{39} \mathrm{H}_{62} \mathrm{O}_{14} \mathrm{Na}(\mathrm{M}+\mathrm{Na})^{+}:$777.4032, found: 777.4010 .

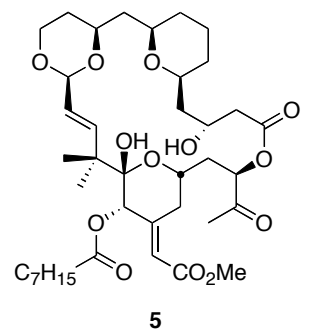

Data for 5:

$\mathbf{R} \boldsymbol{f}$ (4:1 EtOAc: petroleum ether) $=0.85$; one spot, brown (p-anisaldehyde stain).

Optical Rotation: $[\alpha]_{\mathrm{D}}^{23.7}=+2.0\left(\mathrm{c}=0.08, \mathrm{CDCl}_{3}\right)$.

$1 H$ NMR $\left(600 \mathrm{MHz}, \mathrm{C}_{6} \mathrm{D}_{6}\right): \delta=6.40(\mathrm{~d}, J=2 \mathrm{~Hz}, 1 \mathrm{H}), 6.36(\mathrm{~d}, J=16 \mathrm{~Hz}, 1 \mathrm{H}), 5.84(\mathrm{dd}, J=16,7 \mathrm{~Hz}$, $1 \mathrm{H}), 5.67(\mathrm{dd}, J=13,3 \mathrm{~Hz}, 1 \mathrm{H}), 5.68(\mathrm{~s}, 1 \mathrm{H}), 5.62(\mathrm{~s}, 1 \mathrm{H}), 5.32(\mathrm{~d}, J=7 \mathrm{~Hz}, 1 \mathrm{H}), 4.65(\mathrm{~d}, J=12 \mathrm{~Hz}, 1 \mathrm{H})$, 4.53 (ap. t, $J=12 \mathrm{~Hz}, 1 \mathrm{H}), 4.29$ (dd, $J=14,2 \mathrm{~Hz}, 1 \mathrm{H}), 4.19(\mathrm{~m}, 1 \mathrm{H}), 3.86(\mathrm{dd}, J=11.5,4 \mathrm{~Hz}, 1 \mathrm{H}), 3.64$ (ap. t, $J=11.5 \mathrm{~Hz}, 1 \mathrm{H}), 3.57(\mathrm{~m}, 1 \mathrm{H}), 3.19(\mathrm{~s}, 3 \mathrm{H}), 2.90($ ap. t $, J=11 \mathrm{~Hz}, 1 \mathrm{H}), 2.76($ ap. t $, J=11 \mathrm{~Hz}, 1 \mathrm{H})$, 2.42-2.34 (m, 2H), $2.30(\mathrm{dd}, J=13,2.5 \mathrm{~Hz}, 1 \mathrm{H}), 2.09(\mathrm{~m}, 2 \mathrm{H}), 1.90(\mathrm{~m}, 1 \mathrm{H}), 1.71(\mathrm{~s}, 3 \mathrm{H}), 1.66(\mathrm{ddd}, J=$ $15,11.5,8 \mathrm{~Hz}, 1 \mathrm{H}), 1.58-1.42$ (br m, 5H), 1.47 (s, 3H), 1.37 (m, 1H), $1.24(\mathrm{~s}, 3 \mathrm{H}), 1.22-1.16(\mathrm{~m}, 3 \mathrm{H}), 1.13-$ 1.00 (br m, 5H), 0.87-0.78 (br m, 6H), 0.85 (t, $J=7 \mathrm{~Hz}, 3 \mathrm{H}) \mathrm{ppm}$.

${ }^{13} \mathbf{C}$-NMR $\left(150 \mathrm{MHz}, \mathrm{C}_{6} \mathrm{D}_{6}\right): \delta=203.15,171.55,170.57,166.64,152.00,141.89,120.84,103.05,99.85$, $78.30,75.77,75.52,74.81,68.68,66.15,65.01,50.53,45.61,43.10,42.10,39.66,35.35,34.68,32.70$, $31.93,31.55,31.48,31.37,29.23,29.19,25.40,25.01,24.94,23.13,22.90,19.58,14.25 \mathrm{ppm}$.

IR (FTIR, film) v = 3305, 2928, 2849, 1738, 1722, 1666, 1633, 1462, 1434, 1231, 1157, 1139, $1102 \mathrm{~cm}^{-1}$.

HRMS: Calculated for $\mathrm{C}_{39} \mathrm{H}_{60} \mathrm{O}_{13} \mathrm{Na}(\mathrm{M}+\mathrm{Na})^{+}$: 759.3926, found: 759.3912 . 


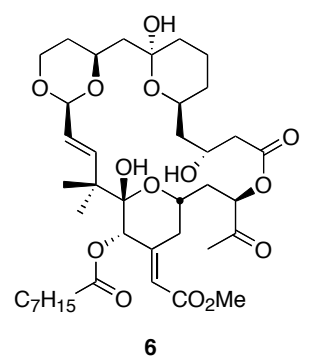

Data for 6 :

$\mathbf{R} \boldsymbol{f}$ (4:1 EtOAc: petroleum ether) $=0.73$; one spot, brown (p-anisaldehyde stain).

Optical Rotation: $[\alpha]_{\mathrm{D}}^{24.0}=+6.2\left(\mathrm{c}=0.1, \mathrm{CDCl}_{3}\right)$.

$1 \mathrm{H}$ NMR $\left(600 \mathrm{MHz}, \mathrm{C}_{6} \mathrm{D}_{6}\right): \delta=6.41(\mathrm{~d}, J=2 \mathrm{~Hz}, 1 \mathrm{H}), 6.35(\mathrm{~d}, J=16 \mathrm{~Hz}, 1 \mathrm{H}), 5.84(\mathrm{dd}, J=16,8 \mathrm{~Hz}$, $1 \mathrm{H}), 5.68(\mathrm{~s}, 1 \mathrm{H}), 5.64(\mathrm{dd}, J=13,3 \mathrm{~Hz}, 1 \mathrm{H}), 5.60(\mathrm{~s}, 1 \mathrm{H}), 5.32(\mathrm{~d}, J=8 \mathrm{~Hz}, 1 \mathrm{H}), 4.55-4.50(\mathrm{~m}, 2 \mathrm{H}), 4.28$ $(\mathrm{dd}, J=13,2 \mathrm{~Hz}, 1 \mathrm{H}), 4.15$ (ap. t $, J=12 \mathrm{~Hz}, 1 \mathrm{H}), 4.00$ (ap. t, $J=10 \mathrm{~Hz}, 1 \mathrm{H}), 3.82$ (dd, $J=11,4.5 \mathrm{~Hz}, 1 \mathrm{H})$, 3.63-3.56 (m, 2H), 3.19 (s, 3H), 2.61 (ap. t, $J=12 \mathrm{~Hz}, 1 \mathrm{H}), 2.36$ (ap. t, $J=13 \mathrm{~Hz}, 1 \mathrm{H}), 2.31$ (dd, $J=12.5$, $2.5 \mathrm{~Hz}, 1 \mathrm{H}), 2.09$ (dt, $J=8,2.5 \mathrm{~Hz}, 2 \mathrm{H}), 1.93-1.85$ (m, 2H), 1.72 (s, 3H), 1.57-1.45 (m, 4H), 1.22-1.04 (br. $\mathrm{M}, 12 \mathrm{H}), 0.87-0.84(\mathrm{~m}, 2 \mathrm{H}), 0.85(\mathrm{t}, J=17 \mathrm{~Hz}, 3 \mathrm{H}), 0.75-0.69(\mathrm{~m}, 2 \mathrm{H}) \mathrm{ppm}$.

${ }^{13}$ C-NMR $\left(150 \mathrm{MHz}, \mathrm{C}_{6} \mathrm{D}_{6}\right): \delta=203.13,171.49,170.88,166.56,151.90,141.97,126.94,120.77,103.09$, $99.79,95.58,74.83,70.52,68.53,67.45,66.05,64.99,50.51,49.04,45.56,41.55,39.40,35.36,34.95$, $34.61,32.77,31.87,31.40,30.84,29.16,29.12,25.28,24.94,24.86,22.83,19.53,18.33,14.18$ ppm.

IR (FTIR, film) $v=3444,3306,2927,2855,1738,1722,1667,1434,1379,1296,1231,1154,1129,1101$, $1013,975 \mathrm{~cm}^{-1}$.

HRMS: Calculated for $\mathrm{C}_{39} \mathrm{H}_{60} \mathrm{O}_{14} \mathrm{Na}(\mathrm{M}+\mathrm{Na})^{+}$: 775.3875, found: 775.3882 .

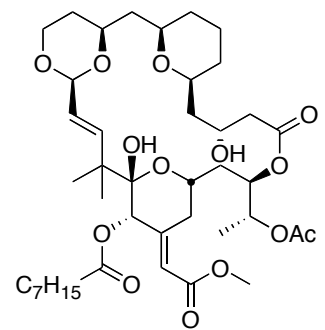

7

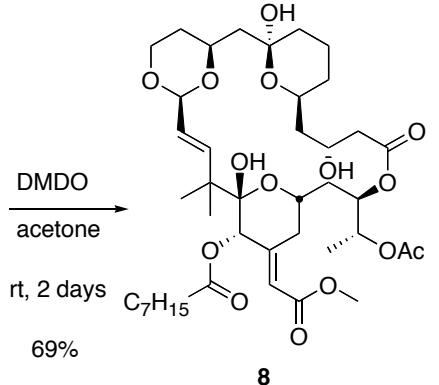

8

To a vial containing acylated nanolog $7(1.6 \mathrm{mg}, 2 \mu \mathrm{mol})$ was added a $0.10 \mathrm{M}$ solution of DMDO in acetone $(0.04 \mathrm{~mL}, 4 \mu \mathrm{mol})$. The vial was briefly flushed with nitrogen and then capped, and the resulting mixture was stirred at ambient temperature for 24 hours. Additional DMDO in acetone $(0.04 \mathrm{~mL}, 4 \mu \mathrm{mol})$ was added, and the resulting solution was stirred for an additional 24 hours. The volatiles were evaporated under a stream of $\mathrm{N}_{2}$. Flash chromatography $\left(\mathrm{SiO}_{2}, 4: 1\right.$ ethyl acetate:pentane) gave C9-oxidized $\mathbf{8}(1.1 \mathrm{mg}$, $69 \%$ ) as a white solid. 


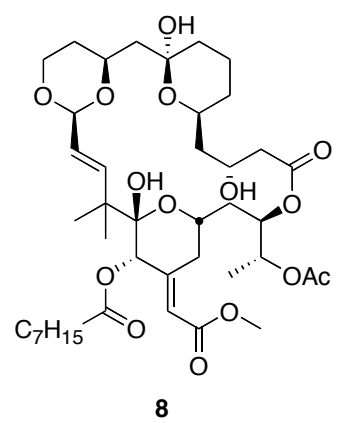

Data for 8:

$\mathbf{R} \boldsymbol{f}(1: 1$ EtOAc: pentane $)=0.23$; one spot, brown (p-anisaldehyde stain).

Optical Rotation: $[\alpha]_{\mathrm{D}}^{22.8}=+3.4\left(\mathrm{c}=0.29, \mathrm{CDCl}_{3}\right)$.

$1 \mathrm{H}$ NMR $\left(500 \mathrm{MHz}, \mathrm{C}_{6} \mathrm{D}_{6}\right): \delta=6.55(\mathrm{~d}, J=16 \mathrm{~Hz}, 1 \mathrm{H}), 6.41(\mathrm{~s}, 1 \mathrm{H}), 5.86(\mathrm{dd}, J=16,7.5 \mathrm{~Hz}, 1 \mathrm{H}), 5.79(\mathrm{~s}$, $1 \mathrm{H}), 5.68$ (brs, $1 \mathrm{H}), 5.66(\mathrm{~m}, 1 \mathrm{H}), 5.56(\mathrm{~d}, J=7.5 \mathrm{~Hz}, 1 \mathrm{H}), 5.22-5.17(\mathrm{~m}, 1 \mathrm{H}), 4.57(\mathrm{~d}, J=7.5 \mathrm{~Hz}, 1 \mathrm{H})$, 4.58-4.45 (m, 1H), $4.37(\mathrm{~d}, J=14 \mathrm{~Hz}, 1 \mathrm{H}) 4.15-4.02(\mathrm{~m}, 2 \mathrm{H}), 3.85(\mathrm{dd}, J=11.5,4 \mathrm{~Hz}, 1 \mathrm{H}), 3.68-3.58(\mathrm{~m}$, $2 \mathrm{H}), 3.20(\mathrm{~s}, 3 \mathrm{H}), 2.59(\mathrm{t}, J=12 \mathrm{~Hz}, 1 \mathrm{H}), 2.44-2.37(\mathrm{~m}, 1 \mathrm{H}), 2.24(\mathrm{dd}, J=12,2.5 \mathrm{~Hz}, 1 \mathrm{H}), 2.07(\mathrm{td}, J=8$, $2.5 \mathrm{~Hz}, 2 \mathrm{H}), 1.92-1.74(\mathrm{~m}, 3 \mathrm{H}), 1.70(\mathrm{~s}, 3 \mathrm{H}), 1.59-1.33(\mathrm{~m}, 9 \mathrm{H}), 1.33-1.02(\mathrm{~m}, 19 \mathrm{H}), 0.92-0.83(\mathrm{~m}, 4 \mathrm{H})$, $0.82-0.67(\mathrm{~m}, 2 \mathrm{H}) \mathrm{ppm}$.

${ }^{13}$ C-NMR $\left(125 \mathrm{MHz}, \mathrm{C}_{6} \mathrm{D}_{6}\right): \delta=171.4,171.1,169.6,166.7,152.3,142.6,120.4,103.1,99.8,95.6,78.7$, $77.7,74.7,70.8,70.5,68.9,67.4,66.1,65.1,50.5,49.0,45.4,41.9,39.8,36.3,35.2,34.6,32.9,31.9,30.9$, $30.1,29.2,26.1,24.9,24.8,22.8,20.7,19.7,18.4,16.4,14.2 \mathrm{ppm}$.

IR (FTIR, film) v = 3368 (brs), 2930 (w), 1742 (s), 1720 (s), 1662 (w), 1436 (w), 1382 (w), 1261 (s), 1233 (s), $1151(\mathrm{w}), 1096(\mathrm{w}), 1020(\mathrm{w}), 804(\mathrm{brs}) \mathrm{cm}^{-1}$.

HRMS: Calculated for $\mathrm{C}_{41} \mathrm{H}_{64} \mathrm{O}_{15} \mathrm{Na}(\mathrm{M}+\mathrm{Na})^{+}:$819.4137, found: 819.4141. 


\section{Spectra}

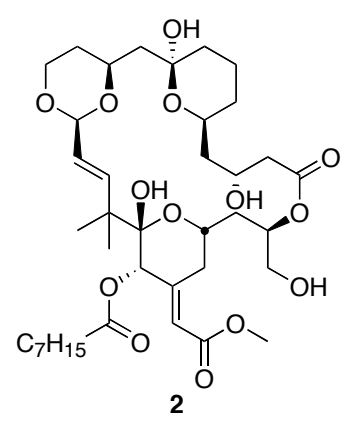

${ }^{1} \mathrm{HNMR}, \mathrm{C}_{6} \mathrm{D}_{6}$

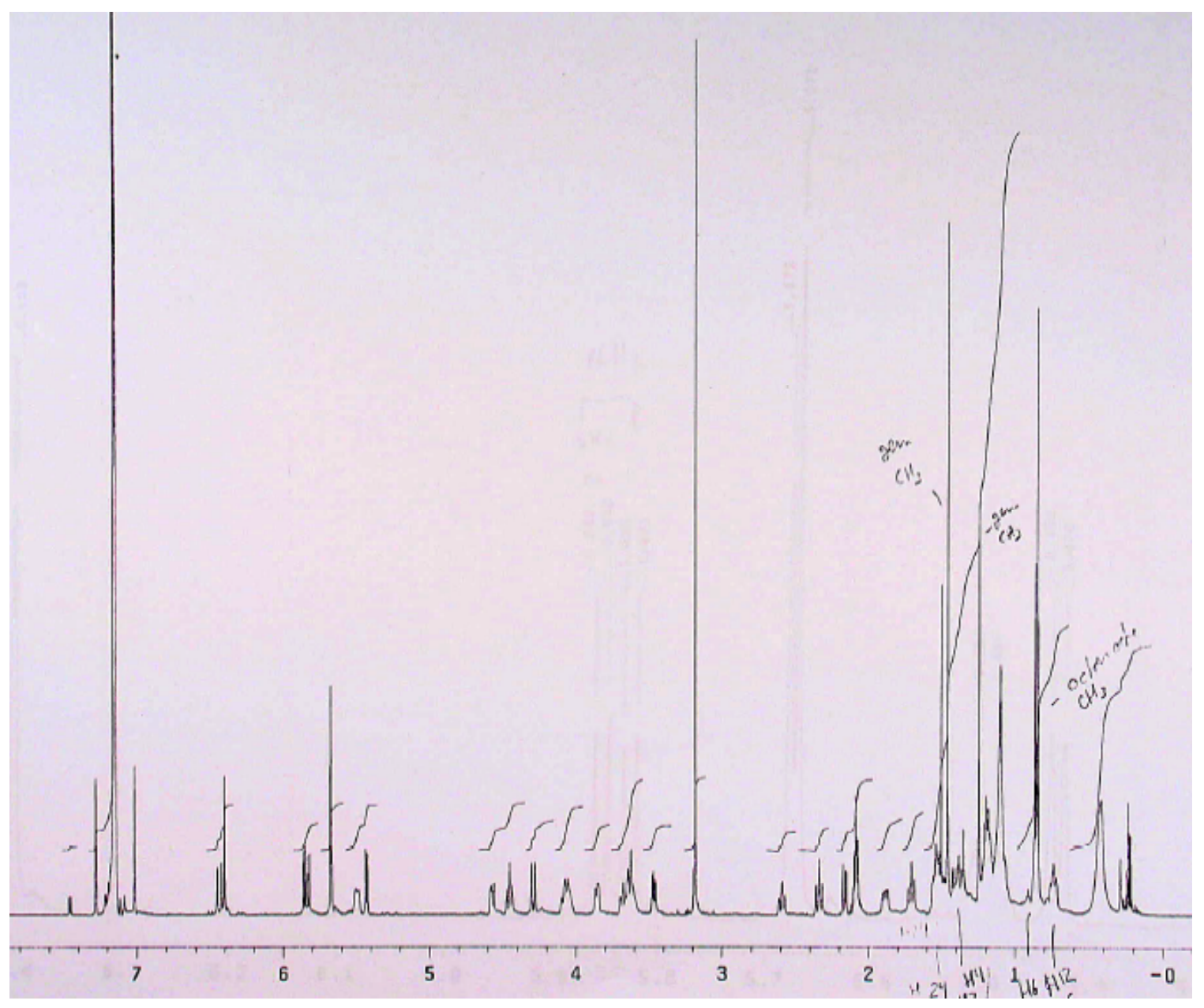




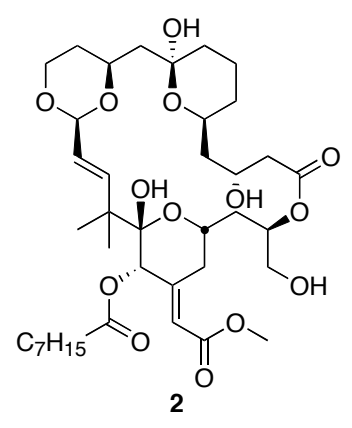

$\operatorname{COSY}, \mathrm{C}_{6} \mathrm{D}_{6}$

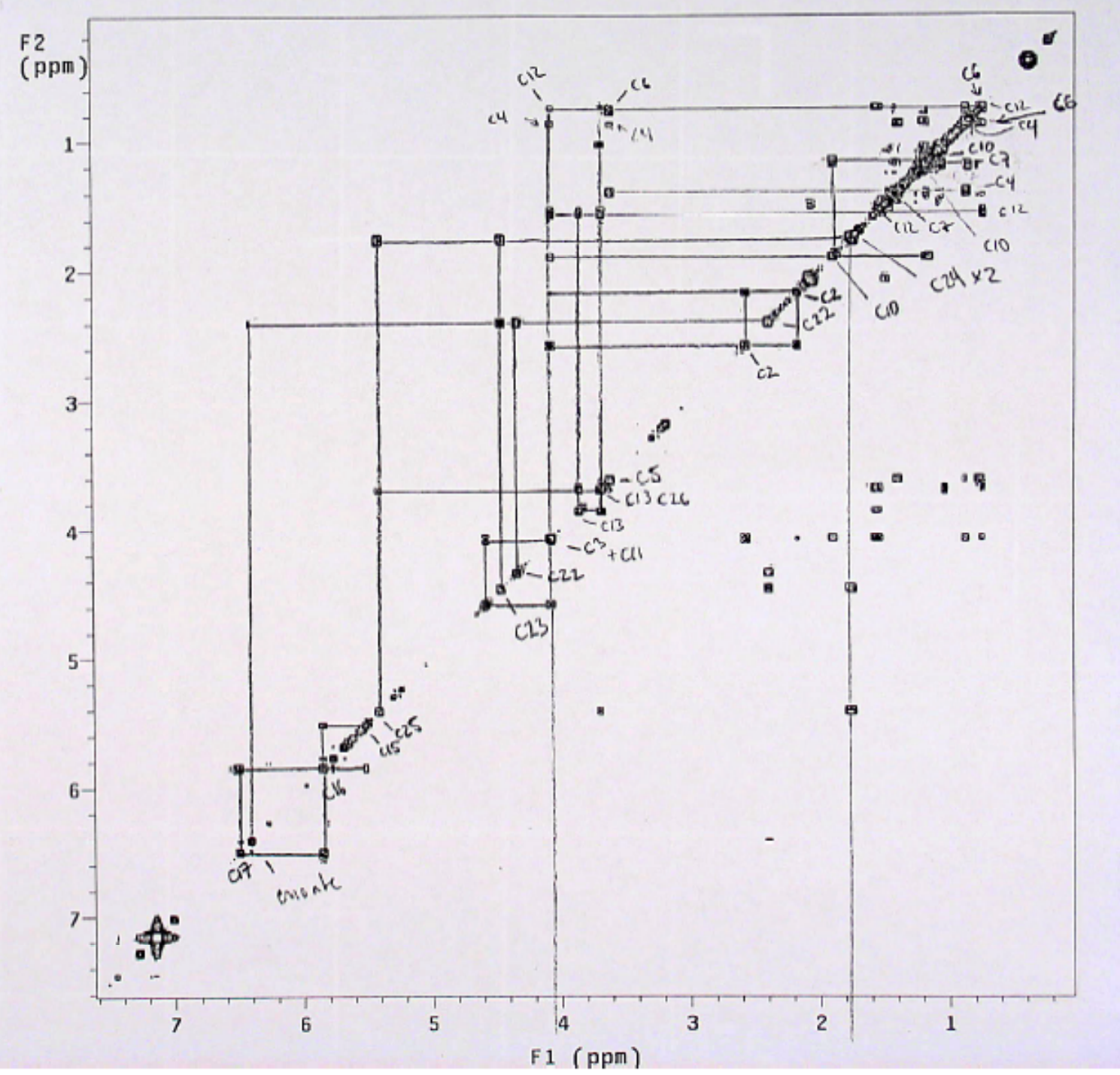




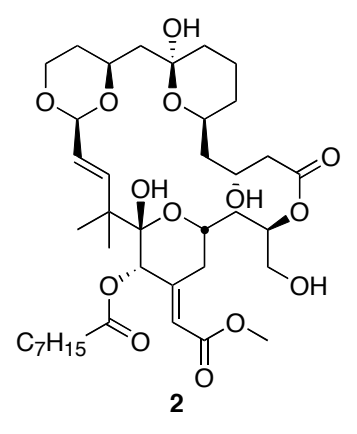

HSQC, $\mathrm{C}_{6} \mathrm{D}_{6}$

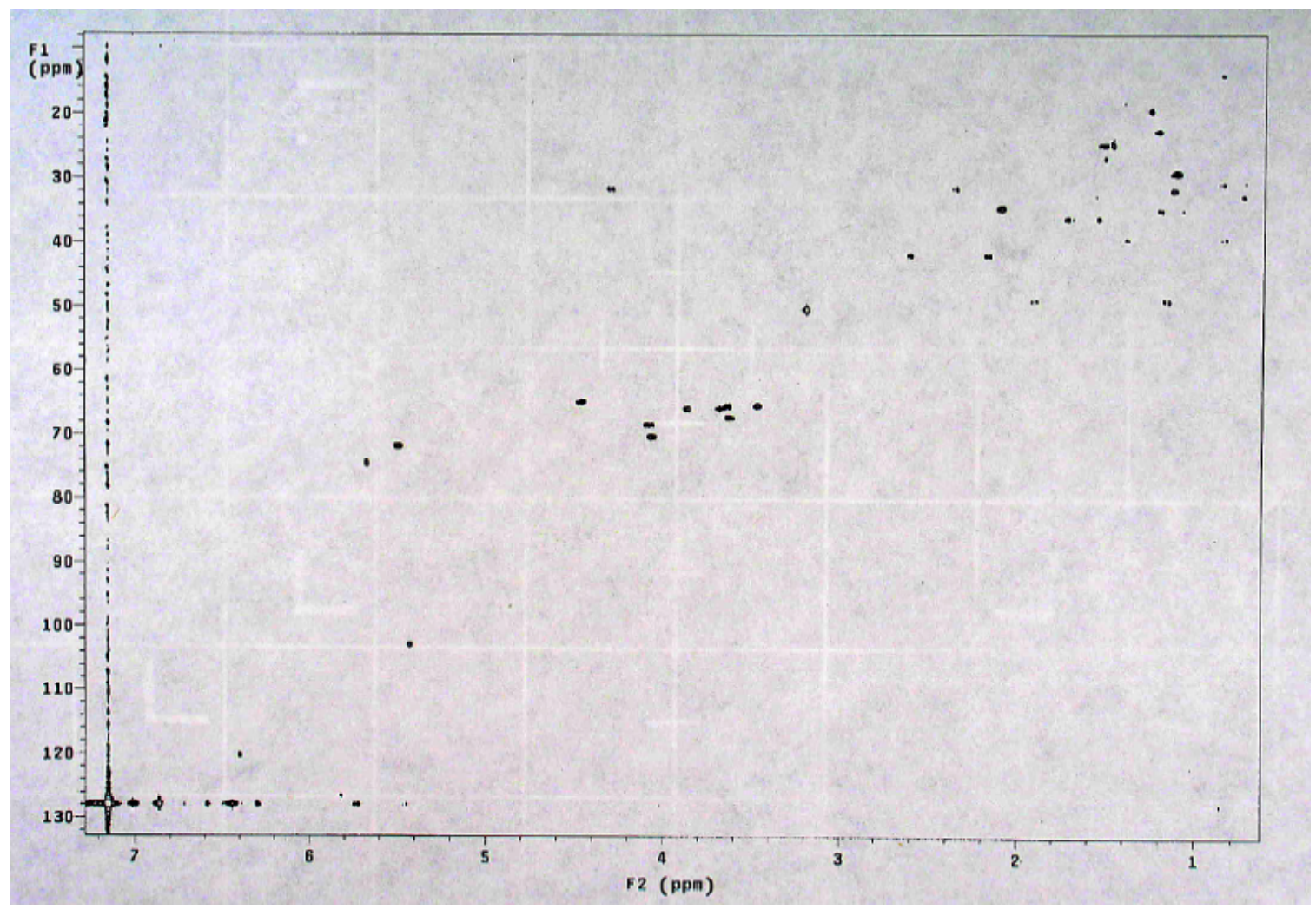

